Bayero Journal of Pure and Applied Sciences, 11(1): 397 - 407

ISSN 2006 - 6996

\title{
EFFECTS OF QUARANTINE ON TRANSMISSION DYNAMICS OF LASSA FEVER
}

\author{
Abdurrahman Abdulhamid ${ }^{(a, b)}$ and Nafiu Hussaini ${ }^{a}$ \\ ${ }^{a}$ Department of Mathematical Sciences, Bayero University Kano, P.M.B. 3011, Kano, Nigeria \\ ${ }^{\mathrm{b}}$ Department of Statistics, Kano State Polytechnic, P.M.B. 3401, Kano, Nigeria. \\ Corresponding author: nhussaini.mth@buk.edu.ng, 08037594137
}

\section{ABSTRACT}

In this paper, a mathematical model of Lassa fever is formulated. The model includes quarantine as a control strategy and allows re-infection. The model is shown to be wellposed. The disease free equilibrium is shown to be locally asymptotically stable whenever the basic reproduction number is less than unity and unstable otherwise. Numerical simulations have been used to show the impact of the control measure.

Keywords: Quarantine, re-infection, immunity, reproduction number, stability.

\section{INTRODUCTION}

Lassa fever, a viral hemorrhagic fever transmitted by rats, is endemic in West Africa (Carey et al., 1972; Frase, 1974; Monath et al., 1973; Monath et al., 1974). After an incubation period of 6 to 21 days, an acute illness with multi-organ involvement develops. Nonspecific symptoms include fever, facial swelling, and muscle fatigue, as well as conjunctivitis and mucosal bleeding. Its symptoms include muscle pain, ulcers of the mucous membranes, headache, internal bleeding and inflammation of the throat. It also causes the destruction of internal tissues; lungs, heart and kidney failure (WHO, 2016). Furthermore, it is an infectious, often fatal, viral disease marked by high fever, which accounts for up to one-third of deaths in hospitals within the affected regions and $10 \%$ to $16 \%$ of total cases (CDC, 2014; CDC, 2015). It kills approximately 5,000 people per year (Richmond and Baglole, 2003).

The recognized human arenavirus infection history in Africa began in 1969 with the death of two medical missionaries mysteriously and the near-fatal illness of a third (Buckley et al., 1970; Frame et al., 1970; Frame, 1975). An arenavirus which was isolated from two of these patients is given the name of Lassa virus after the town of Lassa, Nigeria, where the disease, known as Lassa fever occurred.

Lassa virus is transmitted from animals; specifically it spreads to humans from a rodent known as natal multimammate mouse (Mastomys natalensis) or African rat. This is probably the most common mouse in equatorial Africa, ubiquitous in human households and eaten as a delicacy in some areas (Richmond and Baglole, 2003). Infection in the rodent population is in a persistent asymptomatic state. The virus is probably transmitted by contact with the feces or urine of animals accessing grain stores in residences (Richmond and Baglole, 2003).

The possibility that Lassa virus could be used as a biological weapon has raised the profile of the need for greater understanding of Lassa fever and for more effective control and treatment programmes (Richmond and Baglole, 2003). Because of its high case fatality rate, ability to spread easily by human-to-human contact, and potential for aerosol release, Lassa virus is classified as a Bio-safety Level 4 (BSL4) and NIAID Bio-defense category A agent. The potential use of Lassa virus as a biological weapon directed against civilian or military targets necessitates the development of counter-threat measures, such as diagnostic assays, vaccines and therapeutics. Moreover, the impact of the disease in endemic regions of West Africa is immense, and therefore means to diagnose, treat and prevent this viral hemorrhagic fever will provide a significant public health benefit (CDC, 2015; WHO 2016). Re-infection occurs in Lassa fever as strengthened by (Richmond and Baglole, 2003). Many mathematical models have been designed and used to assess the effect of preventive measures on the spread of Lassa virus in a given community. This study extends the works of (James et al., 2015a; James et al., 2015b; Lo lacono et al., 2015) by inter alia,

[i.] Incorporating environmental contribution to the transmission which is not considered in (James et al., 2015a; Lo lacono et al., 2015);

[ii.] The environment is considered to be saturated;

[iii.] Incorporating quarantine as control measure (James et al., 2015b); 
[iv.] Recovered individuals have temporary immunity (Richmond and Baglole, 2003);

[v.] Latency period is incorporated while it was neglected in (James et al., 2015a, James et al., 2015b);

[vi.] Using incidence rate for both human (constant rate was used in (James et al., 2015a) and rodents population (constant rate was used in (James et al., 2015a, James et al., 2015b)).

The paper is organized as follows. The model with quarantine is formulated in Section 2, analyzed in Section 3 and numerical simulation is presented in Section 4.

\section{Model Formulation}

The total human population, $N_{\mathrm{H}}(t)$, is divided into susceptible individuals $S_{\mathrm{H}}(t)$, asymptomatic individuals $E_{\mathrm{H}}(t)$, symptomatic individuals $I_{\mathrm{H}}(t)$ and individuals in quarantine receiving treatment $Q(t)$, so that:

$N_{\mathrm{H}}(t)=S_{\mathrm{H}}(t)+E_{\mathrm{H}}(t)+I_{\mathrm{H}}(t)+Q(t)$.

Whereas, the total population of rodents, at time $t$ denoted by $N_{\mathrm{R}}(t)$, is divided into two compartments for susceptible rodents and infected rodents, such that:

$N_{\mathrm{R}}(t)=S_{\mathrm{R}}(t)+I_{\mathrm{R}}(t)$.

The susceptible population with risk of Lassa virus infection $S_{\mathrm{H}}(t)$ is generated by recruitment of humans at a constant rate $\Pi$ (all humans recruited into the population are assumed to be at risk of Lassa-infection), infected individuals recover at a rate $\tau_{1}$ and quarantine individuals recover at a rate $\tau_{2}$. The population is decreased by infection at a rate $\lambda_{\mathrm{H}}$, moving from the susceptible class to exposed class at a rate $\lambda_{H}$ and natural death at a rate $\mu_{\mathrm{H}}$. Thus,

$\frac{d S_{H}}{d t}=\Pi+\tau_{1} I_{\mathrm{H}}+\tau_{2} Q-\lambda_{\mathrm{H}} S_{\mathrm{H}}-\mu_{\mathrm{H}} S_{\mathrm{H}}$.

The population of asymptomatic humans $E_{\mathrm{H}}$ is generated by Lassa infection at the rate $\lambda_{H}$. It is reduced by the development of clinical symptoms of Lassa at a rate $\gamma_{1}$ and natural death at the rate $\mu_{\mathrm{H}}$.

$\frac{d E_{H}}{d t}=\lambda_{\mathrm{H}} S_{\mathrm{H}}-\left(\gamma_{1}+\mu_{\mathrm{H}}\right) E_{\mathrm{H}}$.

The population of symptomatic individuals is increased at the rate $\gamma_{1}$ and diminished by recovery at the rate $\tau_{1}$, quarantine at the rate $\gamma_{2}$, natural death at the rate $\mu_{H}$ and death induced by the disease at a rate $\delta$. Thus, $\frac{d I_{H}}{d t}=\gamma_{1} E_{\mathrm{H}}-\left(\tau_{1}+\gamma_{2}+\delta+\mu_{H}\right) I_{H}$.
The population of quarantine individuals is generated as a result of quarantining the symptomatic individuals at the rate $\gamma_{2}$ and diminished by recovery of individuals in the quarantine at the rate $\tau_{2}$, death induced by the disease $\delta$ and natural death $\mu_{H}$. Thus,

$\frac{d Q}{d t}=\gamma_{2} I_{H}-\left(\tau_{2}+\delta+\mu_{H}\right) Q$.

The population of pathogens $P$ in the environment is generated as a result of shedding from the infected rodents at a rate $\alpha$. It is diminished by natural death of the pathogens, so that

$$
\frac{d P}{d t}=\alpha I_{R}-\mu_{P} P
$$

The population of susceptible rodents $S_{R}$ is assumed to follow a logistic growth rate $b_{L}(1-$ $\frac{N_{R}}{K}$ ), where $b_{L}$ is the maximum rate of growth of rodents and $K>N_{R}$ is the carrying capacity (which is related to availability of food and space). This shows that the growth of rodents is density dependent. The rodents population $S_{R}$ decreased by Lassa fever infection and natural death at the rates $\lambda_{R}$ and $\mu_{R}$, respectively. Therefore,

$\frac{d S_{R}}{d t}=b_{L}\left(1-\frac{N_{R}}{K}\right)-\lambda_{R} S_{R}-\mu_{R} S_{R}$.

The population of infected rodents $I_{R}$ is generated following the infection of susceptible rodents at the rate $\lambda_{R}$ and decreased only because of natural death. Hence,

$\frac{d I_{R}}{d t}=\lambda_{R} S_{R}-\mu_{R} I_{R}$.

The model for the Lassa fever is described by the following system of differential equations while the flow diagram of the model is shown in Figure 1. The parameters and associated variables are presented in tables 1 and 2 .

$\frac{d S_{H}}{d t}=\Pi+\tau_{1} I_{\mathrm{H}}+\tau_{2} Q-\lambda_{\mathrm{H}} S_{\mathrm{H}}-\mu_{\mathrm{H}} S_{\mathrm{H}}$.

$\frac{d E_{H}}{d t}=\lambda_{\mathrm{H}} S_{\mathrm{H}}-\left(\gamma_{1}+\mu_{\mathrm{H}}\right) E_{\mathrm{H}}$.

$\frac{d I_{H}}{d t}=\gamma_{1} E_{\mathrm{H}}-\left(\tau_{1}+\gamma_{2}+\delta+\mu_{H}\right) I_{H}$.

$\frac{d Q}{d t}=\gamma_{2} I_{H}-\left(\tau_{2}+\delta+\mu_{H}\right) Q$.

\[ \frac{d P}{d t}=\alpha I_{R}-\mu_{P} P \]
$\frac{d S_{R}}{d t}=b_{L}\left(1-\frac{N_{R}}{K}\right)-\lambda_{R} S_{R}-\mu_{R} S_{R}$.
$\frac{d I_{R}}{d t}=\lambda_{R} S_{R}-\mu_{R} I_{R}$.
where, $\lambda_{H}=\beta_{H} I_{H}+\beta_{E H} \frac{P}{\kappa_{c}+P}+\beta_{R H} I_{R}$
$\lambda_{R}=\beta_{R} I_{R}+\beta_{H R} I_{H}$. and 
- Rodents:

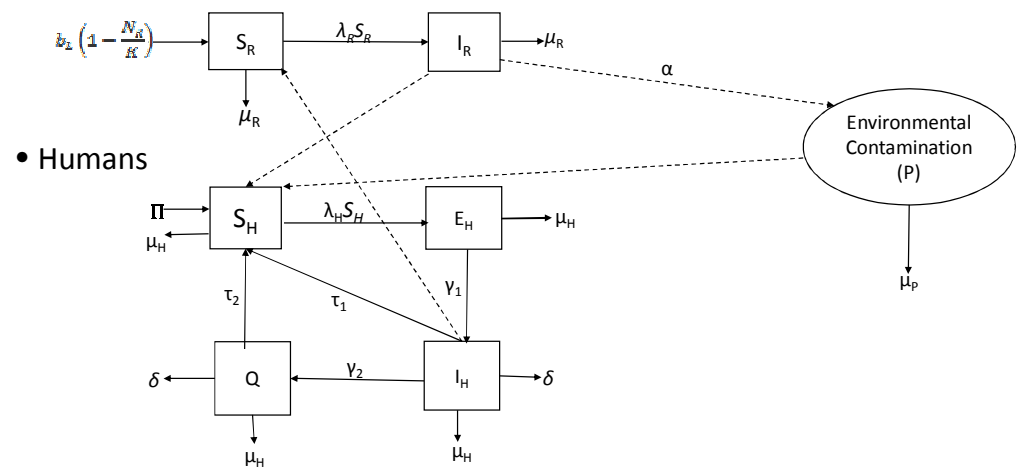

Figure 1: showing the schematic diagram of the model equations 1-7 where solid arrows indicate transitions and dashed arrow indicates interaction. Expressions next to arrows show the per capita flow rate between compartments.

Table 1: Description of the state variables of the model

\begin{tabular}{cl}
\hline Variable & Interpretation \\
\hline$N_{H}$ & Total population of humans \\
$S_{H}$ & Population of susceptible humans with risk of Lassa virus infection \\
$E_{\mathrm{H}}$ & Population of humans exposed to Lassa virus \\
$I_{\mathrm{H}}$ & Population of Lassa-infected humans with symptoms of Lassa fever \\
$\mathrm{Q}$ & Population of individuals in quarantine \\
$N_{\mathrm{R}}$ & Total population of rodents \\
$\mathrm{S}_{\mathrm{R}}$ & Population of susceptible rodents \\
$I_{\mathrm{R}}$ & P population of pathogens in the contaminated environment and air \\
\hline
\end{tabular}

Table 2: Description of parameters of the model.

\begin{tabular}{|c|c|}
\hline Parameter & Interpretation \\
\hline$\Pi$ & Recruitment rate for humans. \\
\hline$\lambda_{H}$ & Rates of Lassa force of infection in humans. \\
\hline$\lambda_{R}$ & Rates of Lassa force of infection in rodents. \\
\hline$\mu_{H}, \mu_{R}, \mu_{P}$ & Natural death rates of humans, rodents and pathogens, respectively. \\
\hline & Transmission rates from infected rodents to susceptible rodents, rodents \\
\hline$\beta_{R}, \beta_{R H}, \beta_{E H}, \beta_{H}, \beta_{H R}$ & $\begin{array}{l}\text { to susceptible humans, contaminated environment and air to susceptible } \\
\text { humans, infected humans to susceptible humans and from humans to } \\
\text { susceptible rodents, respectively. }\end{array}$ \\
\hline$\alpha$ & Rates of shedding from rodent to environment. \\
\hline$\tau_{1}$ & Recovery rate of infected individuals \\
\hline$\tau_{2}$ & Recovery rate of individuals in quarantine \\
\hline$\gamma_{1}$ & Progression rate of expose humans to infected class \\
\hline$\gamma_{2}$ & Progression rate of infected humans to quarantine class \\
\hline$K$ & Carrying capacity for rodents \\
\hline$\delta$ & Disease-induced death rate for humans \\
\hline$b_{I}$ & Maximum rate of growth of rodents \\
\hline
\end{tabular}

Some of the main assumptions made in the formulation of the model are as follows;

[i.] Homogeneous mixing of the human and rodents populations such that there are equal chances of transmitting the virus. Transmission patterns which are possible includes: rodent-torodent, rodent-to-human, human-to-human, human-to-rodent, environment-to-human and rodent contaminate the environment (Lo lacono et al., 2015); [ii.] Successful treatment against Lassa fever does not guarantee permanent immunity against Lassa re-infection (Richmond and Baglole, 2003);

[iii.] Natural recovery is possible (Ajayi, 2014); [iv.] Infected humans can transmit the disease via human-rodent infection (Lo lacono et al., 2015); 
Special Conference Edition, November, 2018

[v.] The virus does not kill the vector (i.e. they die naturally (James et al., 2015a);

The model ( 1 ) extends the works in (James et al., 2015a; James et al., 2015b; Lo lacono et al., 2015) by inter alia,

[i.] Incorporating environmental contribution to the transmission;

[ii.] The environment is considered to be saturated;

[iii.] Incorporating quarantine as control measure (James et al., 2015b);

[iv.] The contribution of individuals is considered negligible;

[v.] The population of the reservoir (rodents) is divided into susceptible and infected classes;

[vi.] Recovered individuals have temporary immunity (Richmond and Baglole, 2003);

[viii.] Latency period is incorporated while it was neglected in (James et al., 2015a, James et al., 2015b);

[viii.] Using a logistic rate for susceptible rodents (constant rate was used in (James et al., 2015a, James et al., 2015b)); [ix.] Using incidence rate for both human (constant rate was used in (James et al., 2015a) and rodents population (constant rate was used in (James et al., 2015a, James et al., 2015b));

\subsection{Basic properties of the model.}

Here, we first prove that a solution to the initial-value problem of system (1) exists and in fact, the solution is unique.

\section{Theorem 2.1}

Let $\left(S_{H 0}, E_{H 0}, I_{H 0}, Q_{0}, P_{0}, S_{R 0}, I_{R 0}\right) \in \mathbb{R}$ be given.

There exist, $t_{0}$ and continuously differentiable functions $\quad\left(S_{H}(\mathrm{t}), \quad E_{H}(\mathrm{t}), \quad I_{H}(\mathrm{t}), Q(\mathrm{t})\right.$, $\left.P(t), S_{R}(\mathrm{t}), I_{R}(\mathrm{t}):\left[0, t_{0}\right) \rightarrow \mathbb{R}\right)$ such that the ordered heptads $\left(S_{H}(\mathrm{t}), \quad E_{H}(\mathrm{t}), \quad I_{H}(\mathrm{t}), Q(t)\right.$, $\left.P(t), S_{R}(\mathrm{t}), I_{R}(\mathrm{t})\right)$ satisfies model $(l)$ and $\left(S_{H}(\mathrm{t})\right.$, $\left.E_{H}(\mathrm{t}), \quad I_{H}(\mathrm{t}), Q(t), \quad P(t), S_{R}(\mathrm{t}), I_{R}(\mathrm{t})\right)(0)=$ $\left(S_{H O}, E_{H 0}, I_{H O}, Q_{0}, P_{0}, S_{R O}, I_{R O}\right)$.

Proof

The Classical Picard-Lindelof theorem will be utilized to prove the result. Since the system of ordinary differential equations is autonomous, it is enough to show that the function $f: \mathbb{R}^{7} \rightarrow$ $\mathbb{R}^{7}$ is defined by

$$
f(y)=\left[\begin{array}{c}
\Pi+\tau_{1} y_{3}+\tau_{2} y_{4}-\lambda_{H} y_{1}-\mu_{H} y_{1} \\
\lambda_{H} y_{1}-\left(\gamma_{1}+\mu_{H}\right) y_{2} \\
\gamma_{1} y_{2}-\left(\tau_{1}+\gamma_{2}+\delta+\mu_{H}\right) y_{3} \\
\gamma_{2} y_{3}-\left(\tau_{2}+\delta+\mu_{H}\right) y_{4} \\
\alpha y_{7}-\mu y_{5} \\
b_{L}\left(1-\frac{N_{R}}{K}\right)-\lambda_{R} y_{6}-\mu_{R} y_{6} \\
\lambda_{R} y_{6}-\mu_{R} y_{7}
\end{array}\right]
$$

where, $\lambda_{H}=\beta_{H} y_{3}+\beta_{E H} \frac{y_{5}}{\kappa_{C}+y_{5}}+\beta_{R H} y_{7}$ and $\lambda_{R}=\beta_{R} y_{7}+\beta_{H R} y_{3}$ is locally Lipchitz in its $y$ argument. In fact, it is enough to show that the Jacobian matrix where,

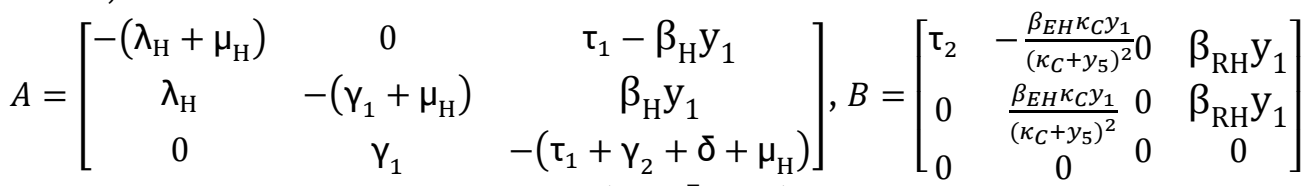

$$
\begin{aligned}
& C=\left[\begin{array}{ccc}
0 & 0 & \mathrm{\gamma}_{2} \\
0 & 0 & \mathrm{a} \\
0 & 0 & 0 \\
0 & 0 & B_{\mathrm{HR}_{6}}
\end{array}\right], D=\left[\begin{array}{cccc}
-\left(\tau_{1}+\delta+\mu_{\mathrm{H}}\right) & 0 & 0 & 0 \\
0 & -\mu_{\mathrm{P}} & 0 & 0 \\
0 & 0 & -\left(\frac{b_{L}}{K}+\lambda_{R}+\mu_{\mathrm{R}}\right) & -\frac{b_{L}}{K}+B_{\mathrm{R}} \mathrm{y}_{6} \\
0 & 0 & \lambda_{R} & -\mu_{\mathrm{R}}+B_{\mathrm{HR}} \mathrm{y}_{6}
\end{array}\right]
\end{aligned}
$$

is linear in $y$ and therefore locally bounded for every $\mathbf{y} \in \mathbb{R}^{7}$ and so $f$ is locally Lipschitz in $y$. By the Picard-Lindelop Theorem, there exists a unique solution, $y(\mathrm{t})$, to the ordinary differential equation $y^{\prime}(t)=f(y(t))$ with initial value $y(0)=y_{0}$ on $\left[0, t_{0}\right]$ for some time $t_{0}>0$. Moreover, for positive initial data it can be shown that solutions remain positive as long as they exist. A lucky by product of the result above is that the obtained solutions are also bounded.
Theorem 2.2 (Boundedness and Positivity).

Suppose the initial conditions of the model (1) satisfy $\left(S_{H O}>0, E_{H O}>0, I_{H O}>0, Q_{0}>0, P_{0}>\right.$ $\left.0, S_{R O}>0, I_{R O}>0\right)$ ). If the unique solution obtained by Theorem 2.1 on the interval $\left[0, t_{0}\right]$ exist for some $t_{0}>0$, then the functions $S_{H}(\mathrm{t})$, $E_{H}(\mathrm{t}), \quad I_{H}(\mathrm{t}), Q(t), \quad P(t), \quad S_{R}(\mathrm{t}), \quad I_{R}(\mathrm{t})$ will be bounded and remain positive for all $t \in\left[0, t_{0}\right]$. 
Proof

Let $S_{H}(\mathrm{t}), E_{H}(\mathrm{t}), I_{H}(\mathrm{t}), Q(t), P(t), S_{R}(\mathrm{t}), I_{R}(\mathrm{t})$ initially have positive values. From Theorem 2.1 there exists at $t^{*}$ such that the solution exists on $\left[0, t^{*}\right]$.

Assuming $T^{*}$ is denoted as the largest time, for which all populations remain positive, that is,

$T^{*}=\sup \left\{t \in\left[0, t^{*}\right]: S_{H}(\mathrm{~s}), E_{H}(\mathrm{~s}), I_{H}(\mathrm{~s}), Q(s), P(s), S_{R}(\mathrm{~s}), I_{R}(\mathrm{~s})>0, \forall s \in\left[0, t_{0}\right]\right\}$

Now on the interval $\left[0, T^{*}\right]$ we can estimate the population values knowing that all constants in the system are positive. Using this and the positivity of solutions on $\left[0, T^{*}\right]$, we can place lower bounds on $\frac{d E_{H}}{d t}, \frac{d I_{H}}{d t}, \frac{d Q}{d t} \cdot \frac{d P}{d t}, \frac{d I_{R}}{d t}$ since

$\frac{d E_{H}}{d t}=\lambda_{\mathrm{H}} S_{\mathrm{H}}-\left(\gamma_{1}+\mu_{\mathrm{H}}\right) E_{\mathrm{H}} \geq-\left(\gamma_{1}+\mu_{\mathrm{H}}\right) E_{\mathrm{H}}$

$\frac{d I_{H}}{d t}=\gamma_{1} E_{\mathrm{H}}-\left(\tau_{1}+\gamma_{2}+\delta+\mu_{H}\right) I_{H} \geq-\left(\tau_{1}+\gamma_{2}+\delta+\mu_{H}\right) I_{H}$

$\frac{d Q}{d t}=\gamma_{2} I_{H}-\left(\tau_{2}+\delta+\mu_{H}\right) Q \geq-\left(\tau_{2}+\delta+\mu_{H}\right) Q$

$\frac{d P}{d t}=\alpha I_{R}-\mu_{P} P \geq-\mu_{P} \mathrm{P}$

$\frac{d I_{R}}{d t}=\lambda_{R} S_{R}-\mu_{R} I_{R} \geq-\mu_{R} I_{R}$

Using separation of variables the populations above at $t \in\left[0, T^{*}\right]$ have their solutions obtained respectively as

$E_{H}(t) \geq E_{H}(0) e^{-\left(\gamma 1+\mu_{H}\right) t}$,

$I_{H}(t) \geq I_{H}(0) e^{-\left(\tau_{1}+\gamma_{2}+\delta+\mu_{H}\right) t}$,

$Q(t) \geq Q(0) e^{-\left(\tau_{2}+\delta+\mu_{H}\right) t}$,

$P(t) \geq P(0) e^{-\mu_{p} t}$

$I_{R}(t) \geq I_{R}(0) e^{-\mu_{R} t}$

Similarly, we can place upper bound on $\frac{d S_{H}}{d t}$ and $\frac{d S_{R}}{d t}$ so that

$\frac{d S_{H}}{d t}=\Pi+\tau_{1} I_{\mathrm{H}}+\tau_{2} Q-\lambda_{\mathrm{H}} S_{\mathrm{H}}-\mu_{\mathrm{H}} S_{\mathrm{H}} \leq \Pi+\tau_{1} I_{\mathrm{H}}+\tau_{2} Q$

$S_{H}(\mathrm{t}) \leq c_{1}(1+\mathrm{t})$, where the constant $\quad c_{1}$ satisfies $c_{1} \geq \max \left[\Pi, K_{1}\right], \quad K_{1}=S_{H}(0)+\frac{\tau_{1} I_{H}(0)}{\left(\tau_{1}+\gamma_{2}+\delta+\mu_{H}\right)}+$ $\frac{\tau_{2} Q(0)}{\left(\tau_{2}+\delta+\mu_{H}\right)}$.

$\frac{d S_{R}}{d t}=b_{L}\left(1-\frac{N_{R}}{K}\right)-\lambda_{R} S_{R}-\mu_{R} S_{R} \leq b_{L}\left(1-\frac{N_{R}}{K}\right) \leq b_{L}$

$S_{R}(\mathrm{t}) \leq S_{R}(0)+b_{L} t$,

$S_{R}(\mathrm{t}) \leq C_{2}(1+\mathrm{t})$, where the constant $C_{2}$ satisfies $C_{2} \geq \max \left[b_{L}, S_{R}(0)\right]$

Now, summing some of the equations $\frac{d E_{H}}{d t}, \frac{d I_{H}}{d t}, \frac{d P}{d t}, \frac{d I_{R}}{d t}$ and place bounds on this sum, we have

$$
\frac{d\left(E_{H}+I_{H}+P+I_{R}\right)}{d t} \leq \lambda_{H} S_{H}+\gamma_{1} E_{H}+\alpha I_{R}+\lambda_{R} S_{R}
$$

There is a bound on $S_{H}$ and $S_{R}$, simplifying after substituting $\lambda_{H}$ and $\lambda_{R}$ we have,

$\frac{d\left(E_{H}+I_{H}+P+I_{R}\right)}{d t} \leq C_{3}(1+t) I_{H}+\left(C_{4}(1+t)+\alpha\right) I_{R}+\gamma_{1} E_{H}+\beta_{E H} P$,

where the constants $C_{3}$ and $C_{4}$ are, respectively, $\left(C_{1} \beta_{H}+C_{2} \beta_{H R}\right)$ and $\left(C_{1} \beta_{R H}+C_{2} \beta_{R}\right)$ also $\left(\kappa_{c}+P>\right.$ $P$ ), $C_{5}$ satisfies $C_{5} \geq \max \left[C_{4}, \alpha\right]$ and $C_{6}$ satisfies $C_{6} \geq \max \left[C_{3}, C_{5}, \gamma_{1}, \beta_{E H}\right]$. Consequently, the inequality becomes

$\frac{d\left(E_{H}+I_{H}+P+I_{R}\right)}{d t} \leq C_{6}(1+t)\left(E_{H}+I_{H}+P+I_{R}\right)$

$\left(E_{H}+I_{H}+P+I_{R}\right)(t) \leq C_{7} e^{t^{2}}$

for $t \in\left[0, T^{*}\right]$ where $C_{7}>0$ depends on $C_{6}>0, E_{H 0}, I_{H 0}, P_{0}, S_{R O}$ and $I_{R 0}$ only. Since $E_{H}(t)$ is positive, we can place an upper bound on $I_{H}(t), P(t), I_{R}(t)$

$C_{7} e^{t^{2}} \geq\left(E_{H}+I_{H}+P+I_{R}\right) \geq I_{H}(t)$

$C_{7} e^{t^{2}} \geq\left(E_{H}+I_{H}+P+I_{R}\right) \geq P(t)$

$C_{7} e^{t^{2}} \geq\left(E_{H}+I_{H}+P+I_{R}\right) \geq I_{R}(t)$

Moreover, since $I_{H}(t), P(t)$ and $I_{R}(t)$ are positive it follows that $E_{H}(t)$ is as well, hence

$C_{7} e^{t^{2}} \geq\left(E_{H}+I_{H}+P+I_{R}\right) \geq E_{H}(t)$

Furthermore, adding the first four equations gives:

$\frac{d N_{H}}{d t}=\Pi-\mu_{H} N_{H}-\delta\left(I_{H}+Q\right)$

Then, $\quad \Pi-\mu_{H} N_{H}-\delta\left(I_{H}+Q\right) \leq \frac{d N_{H}}{d t} \leq \Pi-\mu_{H} N_{H}$

so that 
$\frac{\Pi}{\mu_{H}+\delta} \leq \lim _{t \rightarrow \infty} \inf N_{H}(t) \leq \lim _{t \rightarrow \infty} \sup N_{H}(t) \leq \frac{\Pi}{\mu_{H}}$

This implies that, $N_{H}(t)$ is bounded which shows the individual classes including $Q(t)$ is bounded. Hence, $S_{H}(t)$ and $S_{R}(t)$ can be examined and bound each from below, using

$\frac{d S_{H}}{d t}=\Pi+\tau_{1} I_{\mathrm{H}}+\tau_{2} Q-\lambda_{\mathrm{H}} S_{\mathrm{H}}-\mu_{\mathrm{H}} S_{\mathrm{H}} \geq-\lambda_{H} S_{H}-\mu_{H} S_{H}$.

Substituting $\lambda_{\mathrm{H}}$ and the bound on $I_{H}(t), P(t), I_{R}(t)$ that is, $e^{t^{2}}$, it becomes

$\frac{d S_{H}}{d t} \geq-C_{9}\left(1+e^{t^{2}}\right) S_{H}$

for $t \in\left[0, T^{*}\right]$ where $C_{9}$ satisfies $C_{9} \geq \max \left[\mu_{H}, C_{8}\right]$ and $C_{8} \geq \max \left[\beta_{H}, \beta_{E H}, \beta_{R}\right]$, so

$\frac{d S_{H}}{d t}+C_{9}\left(1+e^{t^{2}}\right) S_{H} \geq 0$. It is known that $\frac{d}{d t}\left(S_{H}(t)+e^{C_{9} \int_{0}^{t}\left(1+e^{\tau^{2}}\right) d \tau}\right) \geq 0$

hence for $t \in\left[0, T^{*}\right], S_{H}(t)>S_{H}(0) e^{-C_{9} \int_{0}^{t}\left(1+e^{\tau^{2}}\right) d \tau}$

Now for $S_{R}(t)$

$\frac{d S_{R}}{d t}=b_{L}\left(1-\frac{N_{R}}{K}\right)-\lambda_{R} S_{R}-\mu_{R} S_{R} \geq-b_{L}\left(\frac{S_{R}+I_{R}}{K}\right)-\lambda_{R} S_{R}-\mu_{R} S_{R}$

$\geq-b_{L}\left(\frac{S_{R}}{K}\right)-b_{L}\left(\frac{I_{R}}{K}\right)-\lambda_{R} S_{R}-\mu_{R} S_{R}$

Substituting $\lambda_{R}$ with the bounds established earlier on $I_{H}$ and $I_{R}$, gives

$\frac{d S_{R}}{d t} \geq-C_{12}\left(1+e^{t^{2}}\right) S_{R}$, for $t \in\left[0, T^{*}\right]$

where $C_{12}$ satisfies $C_{12} \geq \max \left[C_{10}, C_{11}\right]$, and $C_{10}=\left(\frac{b_{L}}{K}+\mu_{R}\right), C_{11}=C_{7} \beta_{R}+C_{8} \beta_{H R}$

hence, $\frac{d S_{R}}{d t}+C_{12}\left(1+e^{t^{2}}\right) S_{R} \geq 0$ and it follows that

$\frac{d}{d t}\left(S_{R}(t)+e^{C_{12} \int_{0}^{t}\left(1+e^{\tau^{2}}\right) d \tau}\right) \geq 0$ hence for $t \in\left[0, T^{*}\right]$,

$S_{R}(t)>S_{R}(0) e^{-C_{12} \int_{0}^{t}\left(1+e^{\tau^{2}}\right) d \tau}$

Thus the values of $S_{H}, E_{H}, I_{H}, Q, P, S_{R}$ and $I_{R}$ remain strictly positive for all $\left[0, T^{*}\right]$, including at time $T^{*}$. By continuity, there must exist a $t>T^{*}$ such that $S_{H}, E_{H}, I_{H}, Q, P, S_{R}$ and $I_{R}$ are still positive.
This
contradicts
the
definition
of
$T^{*}$

$\left(T^{*}=\sup \left\{t\left[0, t^{*}\right]: S_{H}(\mathrm{~s}), E_{H}(\mathrm{~s}), I_{H}(\mathrm{~s}), Q(s), P(s), S_{R}(\mathrm{~s}), I_{R}(\mathrm{~s})>0, \forall s \in\left[0, t_{0}\right]\right\}\right)$

and shows that the model (1) are strictly positive on the entire interval $\left[0, t^{*}\right]$. Furthermore, on the same interval, all of the functions remain bounded, so the interval of existence can be extended further. In fact, the bounds on $S_{H}, E_{H}, I_{H}, Q, P, S_{R}$ and $I_{R}$ derived above hold on a compact time interval. Thus, the time interval may be extended on which the solution exists to $\left[0, t_{0}\right]$ for any $t_{0}>0$ and from the above argument, the solutions remain bounded and positive on $\left[0, t_{0}\right]$.

With this, a general idea that the model is sound was obtained and can stay with certainty that it remains biologically valid as long as it begins with biologically-reasonable (i.e, positive) initial data. Following (Hethcote, 2000), the model is mathematically well-posed and epidemiologically realistic, since all the variables remain nonnegative for all $t>0$. Hence, it is sufficient to consider the dynamics of the model (1) in $D$.

\section{Lemma 2.3.}

The following biologically feasible region of the model equation (1)

$D=\left(S_{H}, E_{H}, I_{H}, Q, P, S_{R}, I_{R} \in \mathbb{R}^{+7}: S_{H}+E_{H}+I_{H}+\right.$ $\left.Q \leq \frac{\Pi}{\mu_{\mathrm{H}}}: S_{R}+I_{R} \leq \frac{b_{L}}{\mu_{\mathrm{R}}}\right)$

is positively invariant and attracting.
Proof. It follows from the fact that $\frac{d N_{H}(t)}{d t}=\Pi-\mu_{H} N_{H}(t) \quad$ and $\quad \frac{d N_{R}(t)}{d t}=b_{L}-$ $\left(\frac{b_{L}}{\mathrm{~K}}+\mu_{\mathrm{R}}\right) N_{R}(t) \geq b_{L}-\mu_{\mathrm{R}} N_{R}(t)$

so, that $\frac{d N_{H}(t)}{d t}<0$, and $\frac{d N_{R}(t)}{d t}<0$ if $N_{H}(t)>\frac{\Pi}{\mu_{H}}$ and $N_{R}(t)>\frac{b_{L}}{\mu_{R}}$

Thus, a standard comparison theorem as in (Lakshmikantham et al., 2015) can be used to show that

$N_{H}(t) \leq N_{H}(0) e^{-\mu_{\mathrm{H}}(\mathrm{t})}+\frac{\Pi}{\mu_{\mathrm{H}}}\left(1-\mathrm{e}^{-\mu_{\mathrm{H}}(\mathrm{t})}\right)$ and $N_{R}(t) \leq N_{R}(0) e^{-\mu_{\mathrm{R}}(\mathrm{t})}+\frac{b_{L}}{\mu_{\mathrm{R}}}\left(1-\mathrm{e}^{-\mu_{\mathrm{R}}(\mathrm{t})}\right)$. In particular, $N_{H}(t) \leq \frac{\Pi}{\mu_{\mathrm{H}}}$ and $N_{R}(t) \leq \frac{b_{L}}{\mu_{\mathrm{R}}}$ if $N_{H}(0) \leq \frac{\Pi}{\mu_{\mathrm{H}}}$ and $N_{R}(0) \leq \frac{b_{L}}{\mu_{\mathrm{R}}}$ respectively. Thus, $D$ is positively-invariant.

Furthermore, if $N_{H}(t)>\frac{\Pi}{\mu_{\mathrm{H}}}$ and $N_{R}(t)>\frac{b_{L}}{\mu_{\mathrm{R}}}$ then either the solution enters $D$ infinite time, or $N_{H}(t)$ approaches $\frac{\Pi}{\mu_{\mathrm{H}}}$ and $N_{R}(t)$ approaches $\frac{b_{L}}{\mu_{\mathrm{R}}}$ and the infected variables approaches zero. Here $D$ is attracting (i.e. all solutions in $\mathbb{R}^{+7}$ eventually approach, enter or stay in $D$ ). Hence the model (1) is epidemiologically wellposed in $D$ as in (Hethcote, 2000). 
Special Conference Edition, November, 2018 Analysis of the model

It is instructive, however, to analyze system (1) first of all. This is done below.

Local asymptotic stability of disease-free equilibrium (DFE)

The human-rodent model (1) has a DFE, obtained by setting the right-hand sides of the equations in the model to zero, given by

$\varepsilon_{0 L}=\left(S_{H}{ }^{*}, E_{H}{ }^{*}, I_{H}{ }^{*}, Q^{*}, P^{*}, S_{R}{ }^{*}, I_{R}{ }^{*}\right)=$ $\left(\frac{\Pi}{\mu_{H}}, 0,0,0,0, \frac{b_{L} K}{b_{L}+K \mu_{R}}, 0\right)$.

The linear stability of $\varepsilon_{0 L}$ will be investigated using the next generation operator method on the system (1). The matrices $F$ (for the new infection terms) and $V$ (for the remaining transition terms) associated with the model are given, respectively, by (noting that $S_{H}{ }^{*}=\frac{\Pi}{\mu_{H}}$ and $S_{R}{ }^{*}=\frac{b_{L} K}{b_{L}+K \mu_{R}}$

The $F$ and $V$ matrix were obtained using the notation of (vanden and Watmough, 2002) for the new infection terms and the remaining transfer terms respectively, and used to compute the spectral radius $\mathcal{R}_{0}$ as

$F=\left(\begin{array}{ccccc}0 & \beta_{H} S_{H}{ }^{*} & 0 & \frac{\beta_{E H}}{\kappa_{c}} S_{H}{ }^{*} & \beta_{R H} S_{H}{ }^{*} \\ 0 & 0 & 0 & 0 & 0 \\ 0 & 0 & 0 & 0 & 0 \\ 0 & 0 & 0 & 0 & 0 \\ 0 & \beta_{H R} S_{R}{ }^{*} & 0 & 0 & \beta_{R} S_{R}{ }^{*}\end{array}\right), \mathrm{V}=\left(\begin{array}{ccccc}k_{1} & 0 & 0 & 0 & 0 \\ -\gamma_{1} & k_{2} & 0 & 0 & 0 \\ 0 & -\gamma_{2} & k_{3} & 0 & 0 \\ 0 & 0 & 0 & \mu_{P} & -\alpha \\ 0 & 0 & 0 & 0 & \mu_{R}\end{array}\right)$

$\mathcal{R}_{0}=\frac{X+\sqrt{Y^{2}+Z}}{2}$

Where

$X=\left(\frac{\beta_{H} \gamma_{1}}{k_{1} k_{2}} \frac{\Pi}{\mu_{H}}+\frac{\beta_{R}}{\mu_{R}} \frac{b_{L} K}{b_{L}+K \mu_{R}}\right), Y=\left(\frac{\beta_{H} \gamma_{1}}{k_{1} k_{2}} \frac{\Pi}{\mu_{H}}-\frac{\beta_{R}}{\mu_{R}} \frac{b_{L} K}{b_{L}+K \mu_{R}}\right), Z=\left(\frac{\beta_{H R} \gamma_{1}}{k_{1} k_{2}} \frac{b_{L} K}{b_{L}+K \mu_{R}}\right)\left(\frac{\beta_{R H}}{\mu_{R}} \frac{\Pi}{\mu_{H}}+\frac{\beta_{E H} \alpha}{\kappa_{c} \mu_{P} \mu_{R}} \frac{\Pi}{\mu_{H}}\right)$

$k_{1}=\left(\gamma_{1}+\mu_{H}\right), k_{2}=\left(\tau_{1}+\gamma_{2}+\delta+\mu_{H}\right), k_{3}=\left(\tau_{2}+\delta+\mu_{H}\right)$

The threshold quantity $\mathcal{R}_{0}$ is the basic reproduction number for Lassa fever (Anderson and May, 1982; Anderson and May 1991; Hethcote, 2000). It represents the average number of secondary cases that one infectious human (or rodent) would generate over the duration of the infectious period if introduced into a completely susceptible human (rodent) population.

Lemma 3.1

The DFE $\varepsilon_{0 L}$, of system (1) is locally asymptotically stable (LAS) if $\mathcal{R}_{0}<1$ and unstable if $\mathcal{R}_{0}>1$.

\section{Simulations}

The numerical simulation was conducted using $O D E 15 s$ in-built in MATLAB where averted cases were computed for $\mathcal{R}_{0}<1$ and $\mathcal{R}_{0}>1$, also four graphs were obtained; graph of infected humans with and without quarantine when $\mathcal{R}_{0}=0.8764$ as depicted in Figure 2; graph of averted cases when $\mathcal{R}_{0}<1$ and AVERTION=4.7128 $\times 10^{9}$ labeled Figure 3. Moreover, graph of infected humans with and without quarantine when $\mathcal{R}_{0}=3.1481$ labelled Figure 4 and graph of averted cases, AVERTION $=4.7128 \times 10^{9}$ label Figure 5. The parameter values in table 4 were used to get the figures below. 


\begin{tabular}{|c|c|c|c|}
\hline Parameter & Interpretation & Range/Baseline value & Reference \\
\hline$\Pi$ & Recruitment rate for humans & $64,787,478$ & $\begin{array}{l}\text { (Richmond and } \\
\text { Baglole, 2003) }\end{array}$ \\
\hline$\mu_{H}, \mu_{R}, \mu_{P}$ & $\begin{array}{l}\text { Natural death rates of humans, } \\
\text { rodents and pathogens respectively }\end{array}$ & $\begin{array}{l}0.018182,0.1858 \\
16248.088819\end{array}$ & $\begin{array}{l}\text { (CIA, 2008; Oliff, } \\
\text { 2003; Stephenson, } \\
\text { 1984) }\end{array}$ \\
\hline $\begin{array}{l}\beta_{R} \\
\beta_{R H} \\
\beta_{E H} \\
\beta_{H}\end{array}$ & $\begin{array}{l}\text { Transmission rates from infected } \\
\text { rodents to susceptible rodents, } \\
\text { rodents to susceptible humans, } \\
\text { ingesting pathogens from the }\end{array}$ & $\begin{array}{l}0.43,0.43, \text { Estimate, } 0.4, \\
\text { Estimate. Dimensionless }\end{array}$ & $\begin{array}{l}\text { (Elizabeth et al., } \\
\text { 2014; Elizabeth et } \\
\text { al., 2014; Estimate; } \\
\text { Elizabeth et al., }\end{array}$ \\
\hline $\begin{array}{l}\text { and } \\
\beta_{H R} \text {. }\end{array}$ & $\begin{array}{l}\text { contaminated environment and air } \\
\text { to susceptible humans, infected } \\
\text { humans to susceptible humans and } \\
\text { from humans to susceptible } \\
\text { rodents, respectively, }\end{array}$ & & 2014; Estimate;) \\
\hline$\alpha$ & $\begin{array}{l}\text { Rates of shedding from rodent to } \\
\text { environment }\end{array}$ & $10^{3}-10^{5}(\mathrm{TCID})_{50 / \mathrm{ml}}$ & (McCormick, 1987) \\
\hline$\kappa_{c}$ & $\begin{array}{l}\text { Concentration of the pathogens in } \\
\text { the contaminated environment and } \\
\text { air }\end{array}$ & $10^{3}-10^{5}(\mathrm{TCID})_{50 / \mathrm{ml}}$ & (McCormick, 1987) \\
\hline$\tau_{1}$ & $\begin{array}{l}\text { Re-infection rate of humans from } \\
\text { infected }\end{array}$ & {$[0.01,0.18]$} & $\begin{array}{l}\text { (Richmond and } \\
\text { Baglole, 2003) }\end{array}$ \\
\hline$\tau_{2}$ & $\begin{array}{l}\text { Re-infection rate of humans from } \\
\text { isolated humans }\end{array}$ & {$[0.01,0.18]$} & $\begin{array}{l}\text { (Richmond and } \\
\text { Baglole, 2003) }\end{array}$ \\
\hline$\gamma_{1}$ & $\begin{array}{l}\text { Progression rate of expose humans } \\
\text { to infected class }\end{array}$ & 0.7869 & $\begin{array}{l}\text { (Richmond and } \\
\text { Baglole, 2003) }\end{array}$ \\
\hline$\gamma_{2}$ & $\begin{array}{l}\text { Progression rate of infected } \\
\text { humans to isolated class }\end{array}$ & {$[0.05,0.08]$} & (McCormick, 1987) \\
\hline K & Carrying capacity for rodents & 946 & (McCormick, 1987) \\
\hline$\delta$ & $\begin{array}{l}\text { Disease-induced death rate for } \\
\text { humans }\end{array}$ & {$[0.0452,0.1133]$} & $(\mathrm{NCDC}, 2018)$ \\
\hline$b_{L}$ & $\begin{array}{l}\text { Maximum rate of growth of rodent } \\
\text { population }\end{array}$ & $\begin{array}{l}1.502 \text { per head } \\
\text { per } 28 \text { days }\end{array}$ & (Oliff, 1953) \\
\hline
\end{tabular}


Special Conference Edition, November, 2018

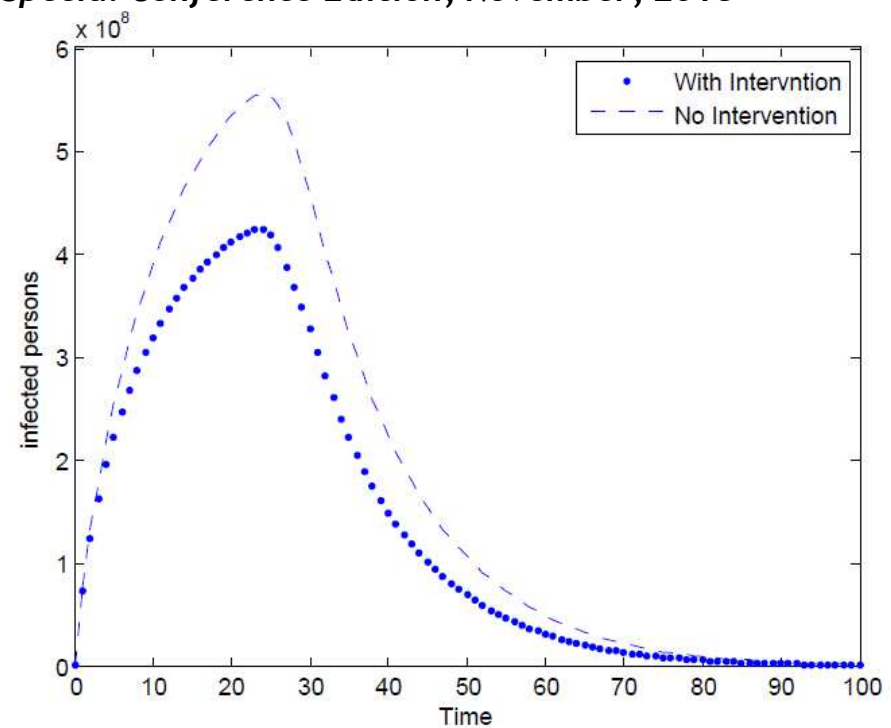

Figure 2. Graph of infected humans with and without intervention when $\mathcal{R}_{0}<1$ at $\beta_{H}=4 \times 10^{-11}$

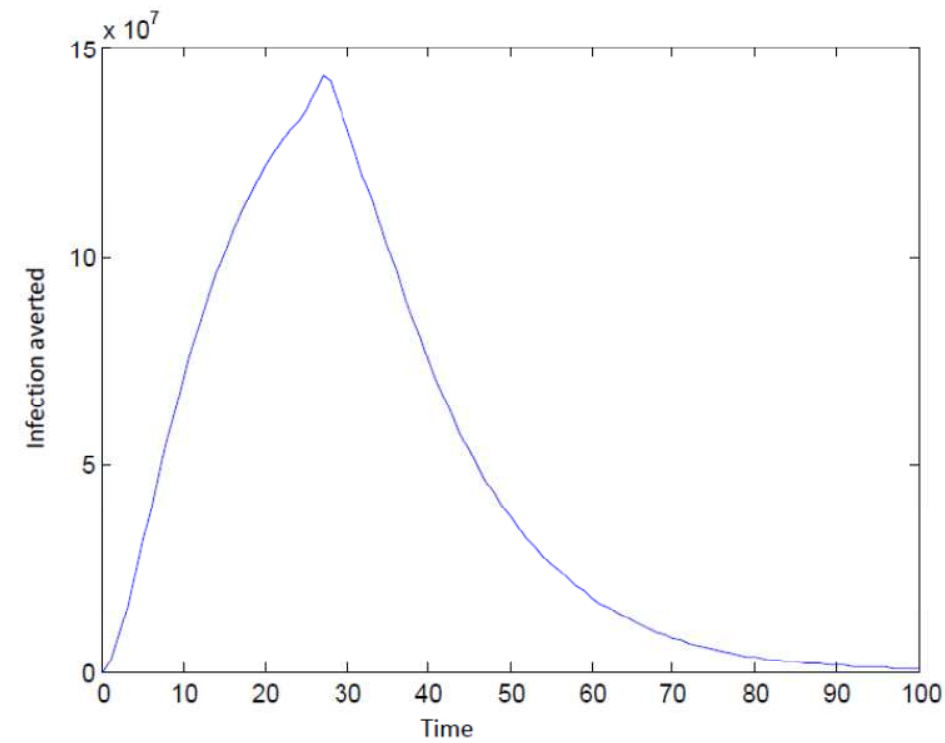

Figure 3. Graph of averted cases, AVERTION $=\mathbf{4 . 7 1 2 8} \times 10^{9}$

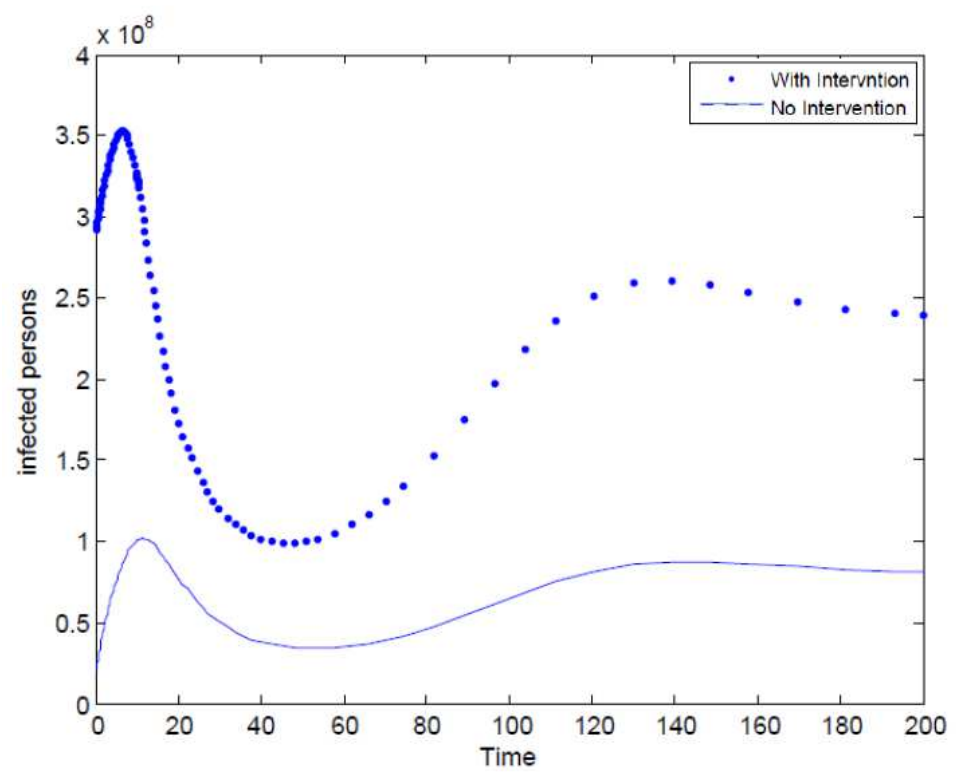

Figure 4. Graph of infected humans with and without quarantine when $\boldsymbol{R}_{\mathbf{0}}>1$ at $\beta_{H}=4 \times 10^{-14}$ 


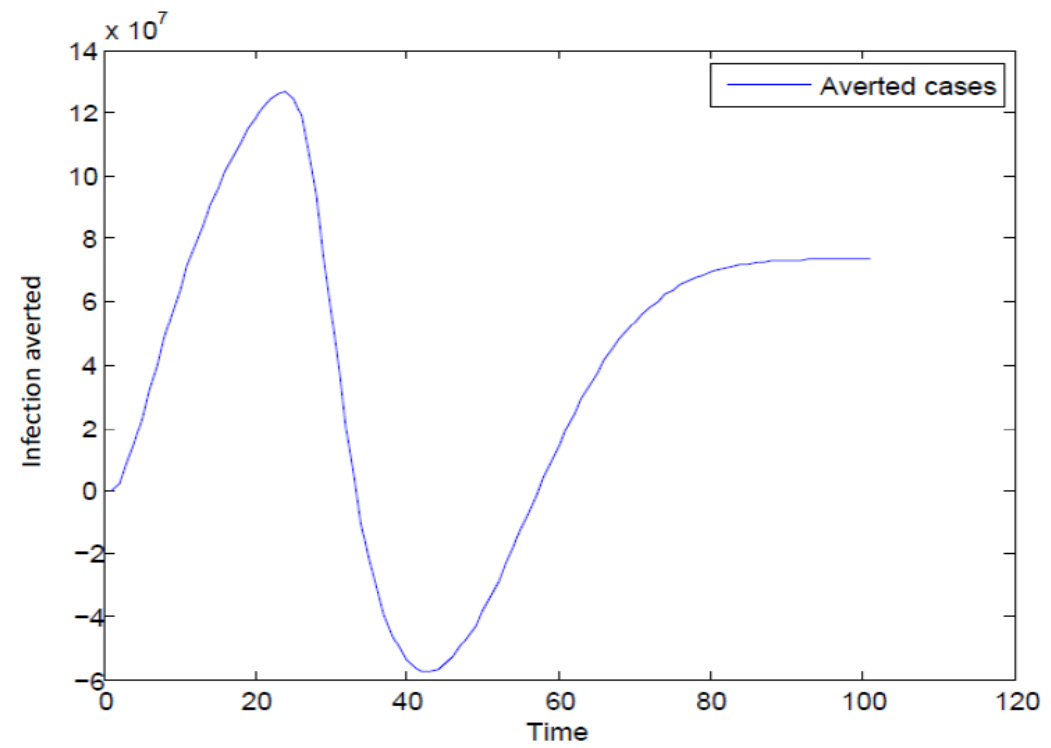

Figure 5. Graph of averted cases, AVERTION $=4.7128 \times 10^{9}$

\section{CONCLUSION}

In this work, a mathematical model is developed and analyzed to study the transmission and control of Lassa fever. Mathematically we modeled Lassa fever as 7dimensional system of non-linear ordinary differential equation. We first show that there exists a domain where our model is well posed mathematically and epidemiologically. The model incorporates quarantine and re-infection parameters. The DFE point of the model is obtained and analyzed for stability. We obtained an important threshold parameter called basic reproductive number $\mathcal{R}_{0}$.

\section{REFERENCES}

Ajayi, N.A., Ukwaja1 K.N., Ifebunandu, N.A., Nnabu, R., Onwe, F.I., Asogun D.A., (2014) Lassa fever-recovery without ribavarin treatment: a case report. African Health Sciences 14 (4) (2014) 1074-1077.

Anderson, R.M., May, R.M., (1991). Infectious Diseases of Humans: Dynamics and Control. Second ed., Oxford University Press, 1991.

Anderson, R.M., May, R.M., (1982). Population Biology of Infectious Diseases. SpringerVerlag, Berlin, Heilderberg, New York, 1982.

Buckley, S.M., Casals, J., Downs, W.G., (1970). Isolation and antigenic characterization of Lassa virus. Nature, 227(5254)(1970) 174-174.

Carey, D.E., Kemp, G.E., White, H.A., Pinneo, L., Addy, R.F., ALMO, F., Stroh, G., Casals J., Henderson B.E., (1972). Lassa fever: epidemiological aspects of the
Numerical simulations, using the parameter values in table 4 show that the associated reproduction number $\mathcal{R}_{0}<1$, and decrease when quarantine is implemented as shown in Figure 2. Thus, the outlook of the effective control of Lassa virus is greatly enhanced if a control strategy based on using quarantine of the infected and infectious human is implemented, which shows the averted cases in Figure 3. But when $\mathcal{R}_{0} .>1$, the Figure 4 and Figure 5 shows the infected humans with and without quarantine when $\mathcal{R}_{0}>1$ at $\beta_{H}=4 \times$ $10^{-14}$, and the averted cases respectively.

1970 epidemic, Jos, Nigeria. Transactions of the Royal Society of Tropical Medicine and Hygiene, 66(3)(1972) 402-408.

Centers for Disease Control and Prevention, National Center for Emerging and Zoonotic Infectious Diseases (NCEZID), Division of High-Consequence Pathogens and Pathology, (DHCPP), Viral Special Pathogens Branch (VSPB), March 25, 2014.

Center for Disease control and prevention (2015). What you need to know about Lassa Fever. CS257280 June 2, 2015, https://www.cdc.gov/vhf/lassa/pdf/w hat-you-need-to-know-about-lassafactsheet.pdf.

Central Intelligence Agency (2018). World fact book for the year 2017. Retrieved on 20 February 2018 from https://www.cia.gov/library/publicatio ns/the-world-actbook/fields/2066.html 
Fichet-Calvet E.,Becker-Ziaja B., Koivogui, L., Gunther, S., (2014). Lassa Serology in Natural Populations of Rodents and Horizontal Transmission. Vector Borne and Zoonotic Diseases. 2014;14(9):665674. doi:10.1089/vbz.2013.1484.

Frame, J.D., Baldwin, J.M., Gocke, D.J., Troup, J.M., (1970). Lassa fever, a new virus disease of man from West Africa. I. Clinical description and pathological findings. The American journal of tropical medicine and hygiene, 19(4)(1970)670-676.

Frame, J.D., (1975). Surveillance of Lassa fever in missionaries stationed in West Africa\}, Bulletin of the World Health Organization, 52(4-6)(1975) 593.

Fraser, D.W., Campbell, C.C., Monath, T.P., Goff, P.A., Gregg, M.B., (1974). Lassa fever in the eastern province of Sierra Leone, 1970-1972. Epidemiologic studies, The American journal of tropical medicine and hygiene 23(6)(1974) 1131-1139.

Hethcote, H.W., (2000). The Mathematics of infectious diseases. SIAM Rev. 42(4)(2000) 599-653.

James, T.O., Abdulrahman, S., Akinyemi, S. and Akinwade, N.I., (2015). Dynamics transmission of Lassa fever disease. International Journal of Innovation and Research in Educational Sciences Volume 2(1)(2015)2349-5219.

James, T.O., Akinyemi, S.T. and Oluwade, B., (2015). Stability analysis of lassa fever with quarantine and permanent immunity. International Journal of Applied Science and Mathematical Theory 1(8)(2015).

Lakshmikantham, V., Leela, S., Martynyuk, A.A., (1995). Stability Analysis of Nonlinear Systems. Marcel Dekker Inc., New York and Basel, ISBN: 0-8247-80671. Astronomische Nachrichten, 316(1)(1995)67-67.

Lo lacono,G., Cunningham, A.A., Fichet-Calvet, E., Garry, R.F., Grant, D.S., Khan, S.H., Leach, M., Moses, L.M., Schieffelin, J.S., Shaffer, J.G., Webb, C.T., Wood, J.L.N., Anderson, R.M., (2015). Using Modelling to Disentangle the Relative Contributions of Zoonotic and Anthroponotic Transmission: The Case of Lassa fever. PLOS Neglected Tropical Diseases, www.plosntds.org, 9(1)(2015) e3398.
McCormick, J.B., (1978). Epidemiology and Control of Lassa fever. Current Topics in Microbiology and Immunology, Springer-Verlag Berlin Heidelberg 134 (1987) 69-78.

Monath, T.P., Mertens, P.E., Patton, R., Mosur, G.R., Baum, N., Pinneo, L., Gary Jr, G.W., Kissling, R.E., (1973). A hospital epidemic of Lassa fever in Zorzor, Liberia, March-April, 1972. The American journal of tropical medicine and hygiene, 22(6)(1973)773-779.

Monath, T.P., Casals J, M.M., Kissling, R.E., Cacciapuoti, A., (1974).Lassa fever in the eastern province of Sierra Leone, 1970-1972. II. Clinical observations and virological studies on selected hospital cases. The American journal of tropical medicine and hygiene, 23(6)(1974)11401149.

Nigeria Centre for Disease Control, Weekly Epidemiological Report, Available online Feburuary 20, 2018, http://www.ncdc.gov.ng/reports

Oliff, O., (1953).The Mortality, Fecundity and Intrinsic Rate of Natural Increase of the Multimammate Mouse, Rattus (Mastomys) natalensis (Smith) in the Laboratory. Journal of Animal Ecology, 22(2)(1953)217-226. doi:10.2307/1814

Richmond, J.K., Baglole, D.J., (2003). Lassa fever: epidemiology, clinical features, and social consequences. BMJ: British Medical Journal, 327(7426)(2003)12711275. doi: $10.1136 / \mathrm{bmj} .327 .7426 .1271$

Stephenson, EH, Larson, E.W, Dominik, J.W., (1984). Effect of environmental factors on aerosol-induced Lassa virus infection. Journal of medical virology, 14(4) (1984)295-303, doi:10.1002/jmv.1890140402.

Van-den Driessche, P., Watmough, J., (2002) Reproduction numbers and subthreshold endemic equilibria for compartmental models of disease transmission. Elsevier; Mathematical Biosciences180(1)(2002)29-48.

World Health Organisation (2016), Disease outbreak news, 19 February 2016 http://www.who.int/csr/don/27-may2016-lassa-fever-nigeria/en/. 